\title{
ASYMPTOTICS FOR OPEN-LOOP WINDOW FLOW CONTROL
}

\author{
ARTHUR W. BERGER \\ ATET Bell Laboratories \\ Room HO-3H-601 \\ Holmdel, NJ 07730-3030. \\ WARD WHITT \\ ATET Bell Laboratories \\ Room $\mathrm{MH}$-2C-178 \\ Murray Hill, NJ 07974-0636.
}

(Received May 1993; revised October 1993)

\begin{abstract}
An open-loop window flow-control scheme regulates the flow into a system by allowing at most a specified window size $W$ of flow in any interval of length $L$. The sliding window considers all subintervals of length $L$, while the jumping window considers consecutive disjoint intervals of length $L$. To better understand how these window control schemes perform for stationary sources, we describe for a large class of stochastic input processes the asymptotic behavior of the maximum flow in such window intervals over a time interval $[0, T]$ as $T$ and $L$ get large, with $T$ substantially bigger than $L$. We use strong approximations to show that when $T>L>>\log T$ an invariance principle holds, so that the asymptotic behavior depends on the stochastic input process only via its rate and asymptotic variability parameters. In considerable generality, the sliding and jumping windows are asymptotically equivalent. We also develop an approximate relation between the two maximum window sizes. We apply the asymptotic results to develop approximations for the means and standard deviations of the two maximum window contents. We apply computer simulation to evaluate and refine these approximations.
\end{abstract}

Key words: Open-loop Control, Traffic Descriptor, Sliding Window, Jumping Window, Scan Statistic, Strong Approximations, Extreme Values, Asymptotics.

AMS (MOS) subject classifications: $60 \mathrm{~F} 05,60 \mathrm{~F} 15$.

\section{Introduction}

The Hungarian influence on probability theory, and mathematics more generally, seems to be greater than can be accounted for solely by chance. In this paper we pay tribute to an outstanding example (who we also claim as an American), Lajos Takács, on the occasion of his 70 th birthday, by applying some Hungarian probability theory to a problem of interest in the 
design of emerging high-speed communication networks. In particular, we apply strong approximations as in Komlós, Major and Tusnády [9], [12], Csörgő and Révész [6] and Csörgö, Horváth and Steinebach [5] to study open-loop window flow-control schemes. We also refer to relate work by Erdös and Rényi [7].

The most familiar window flow-control scheme is a closed-loop control mechanism. Sources regulate their flow into the system (e.g., packets into a network) by keeping track of the flow that has been transmitted but not yet received, typically using acknowledgements sent back from the receiver to the source. The source stops sending when the unacknowledged flow reaches a specified window size; e.g., see pp. 97 and 429 of Bertsekas and Gallager [2].

In contrast, open-loop window flow-control schemes use only local information. For highspeed communication networks, there is growing interest in open-loop control schemes, because end-to-end delays over long distances can become very large relative to the time significant change in the network state occurs; see Budka [3], Reibman and Berger [14] and Sections 2.2 and 3.3 of Roberts [15]. The idea in the open-loop window flow control is to simply limit the maximum input in any interval (window) of specified length. Our purpose here is to gain a better understanding of the way these open-loop window control schemes perform for stationary sources. To do this, we study open-loop sliding and jumping window control schemes via asymptotics.

Let $I(t)$ represent the input to a system in the interval $[0, t]$. We regard $I(t)$ as random, so that $I \equiv\{I(t): t \geq 0\}$ is a real-valued stochastic process. We think of $I$ as being nondecreasing and integer valued, but neither of these properties are required. Let $T$ be the time period and let $L$ be the window length. For simplicity, assume that $T$ is a multiple of $L$. Let $J \equiv J(L, T, I)$ be the random variable representing the maximum jumping window content as a function of $L, T$, and $I$, i.e.,

$$
J \equiv J(L, T, I)=\max \{I(k L)-I((k-1) L): 1 \leq k \leq T / L\}
$$

and let $S \equiv S(L, T, I)$ be the random variable representing the maximum sliding window content as a function of $L, T$, and $I$, i.e.,

$$
S \equiv S(L, T, I)=\sup \{I(t+L)-I(t): 0 \leq t \leq T-L\} .
$$

In the statistics literature, the sliding window is also called the scan statistic; see Naus [12], [13]. More related literature is cited there.

A trivial consequence of these definitions is the ordering

$$
J(L, T, I) \leq S(L, T, I) \text { w.p.1. }
$$

Moreover, since any interval of length $L$ yielding a maximum for $S$ is contained in two consecutive intervals in $J$, and since the maximum jumping window is at least as big as each of these,

$$
S(L, T, I) \leq 2 J(L, T, I) \text { w.p.1. }
$$

Hence, for any process $I$ and any time interval $[0, T]$, the ratio $J / S$ is a random variable with $0.5 \leq J / S \leq 1$. One purpose here is to develop approximations for this ratio.

In general, the distributions of $J$ and $S$ depend on the distribution of the stochastic process $I$ in a rather complicated way. However, as $T$ and $L$ become large, some statistical regularity emerges. Since it is natural to consider large values of $T$ and $L$ in communication network 
applications, in the remaining sections we discuss asymptotics. We start in $\S 2$ by reviewing the central limit theorem and strong approximations, which justify an approximation by Brownian motion. In $\S 3$ we consider extreme-value asymptotics for Brownian motion as $T \rightarrow \infty$. In $\S 4$ we provide conditions for the Brownian approximation to be asymptotically correct and establish limits for $S$ and $J$ as both $T$ and $L$ get large. In particular, there we develop support for the following relatively simple approximations for the random variables (and their means) provided that $\log T<<L<<T$, where $<<$ means much smaller than:

where

$$
S \approx E S \approx \lambda L+\Phi_{S}(T, L) \sqrt{\lambda c^{2} L}
$$

$$
\begin{gathered}
\Phi_{S}(T, L)=\sqrt{2 \log (T / L)+2 \log \log T}-\frac{1.28}{\sqrt{2 \log (T / L)}} \\
J \approx E J \approx \lambda L+\Phi_{J}(T, L) \sqrt{\lambda c^{2} L}
\end{gathered}
$$

where

$$
\Phi_{J}(T, L)=\sqrt{2 \log (T / L)}-\frac{\log \log (T / L)+1.38}{2 \sqrt{2 \log (T / L)}}
$$

and

$$
\frac{J-\lambda L}{S-\lambda L} \approx \frac{E J-\lambda L}{E S-\lambda L} \approx E\left[\frac{J-\lambda L}{S-\lambda L}\right] \approx \frac{\Phi_{J}(T, L)}{\Phi_{S}(T, L)}
$$

for $\Phi_{S}$ and $\Phi_{J}$ defined above. In (1) $-(3), \lambda$ is the rate and $c^{2}$ is the asymptotic variability parameter of $I$ (defined in (6) below). In (1) - (3) and throughout the paper, log means the natural logarithm, i.e., to the base $e$. Without loss of generality (by the choice of the measuring units), we can let $\lambda=1$. Hence, there are really only three parameters in (1) $-(3): L, T$, and $c^{2}$.

The approximations for $S$ and $J$ in (1) and (2) have a simple interpretation: The first term $\lambda L$ is the approximate mean for a single interval of length $L$; the term $\sqrt{\lambda c^{2} L}$ is the approximate standard deviation for a single interval of length $L$; and the remaining factors $\Phi_{S}(T, L)$ for $S$ in (1) and $\Phi_{J}(T, L)$ for $J$ in (2) represent the increase due to the maximization. It is significant that the factors $\Phi_{S}$ and $\Phi_{J}$ depend only on $T$ and $L$, and not upon $\lambda$ and $c^{2}$.

Note that, by (3), the ratio $(J-\lambda L) /(S-\lambda L)$ approaches 1 as $T \rightarrow \infty$, but (3) provides an approximation for the ratio for finite $T$. This ratio estimate as a function of $L$ and $T$ is displayed in Table 1. We can also use (1) and (2) to develop an approximate expansion directly for the ratio $J / S$ in powers of $1 / \sqrt{\lambda L}$. In particular, by (1) and (2),

$$
\frac{J}{S} \approx \frac{1+\sqrt{\frac{c^{2}}{\lambda L}} \Phi_{J}}{1+\sqrt{\frac{c^{2}}{\lambda L}} \Phi_{S}} \approx 1-\sqrt{\frac{c^{2}}{\lambda L}}\left(\Phi_{S}-\Phi_{J}\right)+\frac{c^{2}}{\lambda L} \Phi_{S}\left(\Phi_{S}-\Phi_{J}\right)
$$

However, we will focus on (3).

The maximum window contents $S$ and $J$ are of course random variables. We also derive rough estimates for the standard deviations, namely,

$$
S D V(S) \approx S D V(J) \approx 1.28 \sqrt{\frac{\lambda c^{2} L}{2 \log (T / L)}} ;
$$


see Section 3. Moreover, we would approximate the distributions of $S$ and $J$, after appropriate normalization, by the type- $I$ extreme-value distribution, i.e., the cdf (cumulative distribution function) $\exp \left(-e^{-x}\right),-\infty<x<\infty$; see p. 4 of Leadbetter, Lindgren and Rootzén [11] and (10) $-(13)$ below.

We can combine (1), (2), and (4) to obtain estimates of the coefficients of variation $(C V$, standard deviation divided by the mean) of $S-\lambda L$ and $J-\lambda L$. Keeping only the dominant $\sqrt{2 \log (T / L)}$ term in $\Phi_{S}$ and $\Phi_{J}$, we obtain

$$
C V(S-\lambda L) \approx C V(J-\lambda L) \approx \frac{0.64}{\log (T / L)} .
$$

Hence, if $T / L=10^{k}$, then $C V(S-\lambda L) \approx 0.278 / k$. If $k \geq 3$, then the standard deviation of $S-\lambda L$ should be less than $10 \%$ of the mean.

In $\S 5$ we briefly discuss discriminating windows with $L=O(\log T)$, for which the asymptotics depend upon more than two parameters $\lambda$ and $c^{2}$, and thus have more potential for providing information about the input process.

In $\S 6$ we make numerical comparisons using computer simulations. In particular, we consider 10 replications each of several renewal processes with $\lambda=1, T=10^{6}$ and various values of $L$. These simulation experiments provide additional support for approximations (1) - (5). Indeed, the last term in $\Phi_{S}$ in (1) was suggested by the simulations. In $\S 7$ we state our conclusions.

We were motivated to look for approximations (1) and (2), because we want to compare the open-loop window flow-control schemes to other open-loop flow-control schemes. In particular, in [1] we compare the sliding window to a leaky-bucket-based flow-control scheme. There we conclude that the sliding window admits larger bursts than the leaky bucket for given peak rate and given sustainable rate. To draw this conclusion, we carry out a specific construction: We generate a sample path of a stationary point process $I$ and specify a window length $L$. The reciprocal of the observed minimum distance between consecutive points is the realized peak rate, and the ratio $S / L$ is the maximum possible sustainable rate, when $S \equiv S(L, T, I)$ is the maximum sliding window content. With this peak rate and sustainable rate, the observed sample path just passes through the sliding window control. We then let the leaky bucket have drain rate $r$ equal to the sustainable rate for the sliding window, and let the bucket capacity be the smallest level such that the observed stochastic sample path just passes through the leaky bucket. With this construction, both controls have the same peak and sustainable rates, and the given sample path just passes through both controls. Finally, with the control parameters so determined, we ask which control scheme allows larger bursts, where a burst is defined as the maximum number of consecutive arrivals at the peak rate. We find that the sliding window allows larger bursts, and we use (1) to estimate the difference. See [1] for more details. Reibman and Berger [14] reach the same conclusions for sample video teleconferencing sequences.

\section{The Central Limit Theorem and Strong Approximations}

First, as $T$ gets large, we can apply extreme value theory, as in Leadbetter, Lindgren and Rootzén [11]. In general, though, the limiting behavior depends quite delicately upon the input process $I$. However, if we can assume that $L$ also is suitably large, then we can hope for significant simplification. As $L$ gets large, in great generality, each increment $I(t+L)-I(t)$ becomes approximately normally distributed by the central limit theorem (CLT), i.e.,

$$
\frac{I(t+L)-I(t)-\lambda L}{\sqrt{\lambda c^{2} L}} \Rightarrow N(0,1) \text { as } L \rightarrow \infty
$$


for all $t$, where $\Rightarrow$ denotes convergence in distribution, $N(0,1)$ denotes a standard (zero mean, unit variance) normal random variable, $\lambda$ is a positive constant called the input rate and $c^{2}$ is a positive constant called the asymptotic variability parameter.

To have the CLT (6), we need to have the second moment $E I(t)^{2}$ finite and we need to have the dependence between the increments $I(t+h)-I(t)$ and $I(s)-I(s-h)$ for $s<t$ and $h>0$ decrease suitably as $t-s$ increases. We assume that we do not have infinite second moments or the long-range dependence that would invalidate (6).

If $I$ is a stationary stochastic point process (i.e., has stationary increments) then $c^{2}$ is typically the limiting value of the index of dispersion for counts (IDC), i.e.,

$$
c^{2}=I_{c}(\infty) \equiv \lim _{t \rightarrow \infty} \frac{\operatorname{Var} I(t)}{E I(t)}
$$

see p. 71 of Cox and Lewis [4]. (The role of the second moment condition is clear in (7).) If $I$ is a renewal counting process with i.i.d interrenewal times $X_{k}$, then $c^{2}$ is the squared coefficient of variation of $(\mathrm{SCV})$ of $X_{1}$, i.e.,

$$
c^{2}=S C V\left(X_{1}\right)=\frac{\operatorname{Var} X_{1}}{\left(E X_{1}\right)^{2}}
$$

(It is important that a square appears in the denominator of (8), but not in (7).)

In fact, we need more than (6), and we often have it. In great generality, a properly normalized input process such as $I$ can be approximated by a Brownian motion. Indeed, our key assumption will be that $I$ can be approximated strongly by a Brownian motion, i.e., there exists a standard (zero drift, unit variance) Brownian motion $(B M) B \equiv\{B(t): t \geq 0\}$ such that

$$
\sup \left\{\left|\frac{I(t)-\lambda t}{\sqrt{\lambda c^{2}}}-B(t)\right|: 0 \leq t \leq T\right\}=O(\log T) \text { w.p.1 as } T \rightarrow \infty
$$

see Csörgö and Révész [6] and Csörgö, Horváth and Steinebach [5]. These references show that (9) holds in great generality, so that this seems to be a reasonable starting point for us. Note that (9) can be regarded as a form of functional central limit theorem (FCLT) generalizing the CLT in (6). To convert (9) into the FCLT, replace $t$ in (9) by $n t, T$ by $n$, and then divide by $\sqrt{n}$. This yields

$$
\sup \left\{\left|\frac{I(n t)-\lambda n t}{\sqrt{\lambda c^{2} n}}-\frac{B(n t)}{\sqrt{n}}\right|: 0 \leq t \leq 1\right\}=O\left(\frac{\log n}{\sqrt{n}}\right) \text { w.p.1 as } n \rightarrow \infty
$$

and note that for each $n,\{B(n t) / \sqrt{n}: t \geq 0\}$ is distributed the same as $\{B(t): t \geq 0\}$. Hence, from (9) we get

$$
\frac{I(n t)-\lambda n t}{\sqrt{\lambda c^{2} n}} \Rightarrow B(t) \text { as } n \rightarrow \infty
$$

with the convergence being in the function space $D[0,1]$; see pp. $11-15$ and $88-95$ of [6] for further discussion.

It is also significant that the error in (9) can be regarded as best possible; see [5], [6]. For a renewal process with interrenewal times $X_{k}$ having mean $\lambda^{-1}$ and SCV $c^{2}$, (9) holds when $E e^{s X_{1}}<\infty$ for some $s>0$; see Corollary 3.1 on p. 1450 of Csörgö, Horváth and Steinebach [5]. (Other strong approximations hold with bigger errors when only $E X_{1}^{p}<\infty$ for some $p>2$; see 
[5], [6].) Theorem 3.1 of [5] also provides a means to treat a large class of counting processes with dependent intervals. Moreover, $I(t)$ need not be a counting process; e.g., it could be the input in a fluid model.

In applications, e.g., to communications networks, we are often interested in large $L$ and large $T$. A key issue in the asymptotics is the way these two parameters grow. We consider this issue in $\S 4$.

\section{Extreme Values for Brownian Motion}

In this section we simply suppose that $I$ can be approximated by a $B M$. Consistent with (9), we assume that $I(t)=\lambda t+\sqrt{\lambda c^{2}} B(t)$, where $B$ is a standard $B M$. In this case, we can apply classical results to describe $J$ and $S$ as $T \rightarrow \infty$ for any fixed $L$, omitting $I$ from the notation. In particular, by the extreme-value theorem for i.i.d. normal random variables in Theorem 1.5.3 on p. 14 of [11],

$$
a(L, T)(J(L, T)-\lambda L-b(L, T)) \Rightarrow Z \text { as } T \rightarrow \infty
$$

where $Z$ is the classical type- $I$ extreme-value distribution with cdf

$$
\begin{gathered}
P(Z \leq x)=\exp \left(-e^{-x}\right),-\infty<x<\infty \\
a(L, T)=\sqrt{2 \log (T / L) / \lambda c^{2} L}
\end{gathered}
$$

and

$$
b(L, T)=\sqrt{\lambda c^{2} L}\left[\sqrt{2 \log (T / L)}-\frac{[\log \log (T / L)+\log 4 \pi]}{2 \sqrt{2 \log (T / L)}}\right] .
$$

Note that $E Z=0.5772$ (Euler's constant), $\operatorname{Var} Z=\pi^{2} / 6=1.645, \quad S C V(Z)=4.94$, $\operatorname{median}(Z)=0.3667$ and $\bmod (Z)=0.9624$ for the type- $I$ extreme-value random variable $Z$ in (10); see Chapter 21 of Johnson and Kotz [8].

We use (10) to develop approximation (2) for the mean of $J$ and thus $J$ itself. In particular, we let

$$
J(L, T) \approx \lambda L+b(L, T)+\frac{E Z}{a(L, T)},
$$

which reduces to (2). We also use (10) to obtain an estimate of the variability of $J$. Based on $(10)$, we have the approximation

$$
S D V(J) \approx \frac{S D V(Z)}{a(L, T)}
$$

which yields (4).

We point out that it is not obvious that approximations (14) and (15) ((2) and (4)) will be good. First, we need to approximate $I$ by Brownian motion and, second, we need the extremevalue asymptotics for Brownian motion to perform suitably well for realistic values of $T$. It is well known that the CLT (6) often yields remarkably good approximations for moderate values of the limiting variable (when there is weak dependence). In contrast, the extreme-value limit 
theorems do not yield such good approximations. In part, this is due to the rate of convergence in the extreme-value theorems being remarkably slow; see Section 2.4 of Leadbetter, Lindgren and Rootzén [11]. For example, for the limit (10) the rate of convergence of the normalized cdf's is slower than $1 / \log (T / L)$; see (2.4.8) and (2.4.10) of [11]. Hence, even if $T=10^{6}$ and $L=10^{3}$, good approximations based on (10) are not automatic. Thus, we should be interested in the computer simulations in $\S 6$.

As a corollary to $(10)$, we can divide by $a(L, T)$ and obtain the simpler result

$$
J(L, T)-\lambda L-\sqrt{2 \lambda c^{2} L \log (T / L)} \Rightarrow 0 \text { as } T \rightarrow \infty .
$$

When we divide by $\sqrt{\log (T / L)}$ in (16), we obtain

$$
\frac{J(L, T)-\lambda L}{\sqrt{\log (T / L)}} \Rightarrow \sqrt{2 \lambda c^{2} L} \text { as } T \rightarrow \infty
$$

for fixed $L$. In (16) and (17) convergence in distribution is equivalent to convergence in probability since the limits are deterministic. Note that (16) and (17) yield cruder approximations than (10).

An analog of (17) also exists for $S$; see Corollary 1.2 .3 on p. 31 of [6] (also using the observation on the bottom of p. 29 and Remark 1.2 .1 on p. 30 of [6]). In particular,

$$
\frac{S(L, T)-\lambda L}{\sqrt{\log (T / L)}} \rightarrow \sqrt{2 \lambda c^{2} L} \text { w.p. } 1 \text { as } T \rightarrow \infty .
$$

The limits (17) and (18) imply that

$$
\frac{S(L, T)-\lambda L}{J(L, T)-\lambda L} \Rightarrow 1 \text { as } T \rightarrow \infty .
$$

Hence, using the Brownian approximation, by (19) $S$ and $J$ are asymptotically equivalent as $T \rightarrow \infty$ for any fixed $L$. Moreover, (17) and (18) are consistent with $S$ and $J$ being asymptotically approximated by (1) and (2), but we need additional refinement to get the approximations in (1) and (2).

\section{The Role of $L$ in the Brownian Motion Approximation}

The asymptotics in the previous section apply if we do indeed have a $B M$ approximation. However, the quality of the Brownian approximation for the increments of another process $I$ depends on $L$ being large. Indeed, for the CLT in (6), we required that $L \rightarrow \infty$.

Moreover, in order to have the Brownian extreme-value theory apply to the process $I$, it is evident that $L$ must go to infinity as $T$ goes to infinity. In particular, we need $L_{T} / \log T \rightarrow \infty$, where the notation $L_{T}$ indicates that $L$ depends on $T$. When $L_{T}=O(\log T)$, the asymptotics for $I$ depend on more than two parameters $\lambda$ and $c^{2}$. The case in which $I(t)$ is a partial sum of i.i.d. random variables is discussed in Sections 2.4 and 3.1 of [6]; also see $\S 5$ below.

When we stipulate that $L_{T}$ grows suitably fast as $T \rightarrow \infty$, we can obtain a positive result. The following parallels Theorem 3.1 .1 on p. 115 of [6].

Theorem 1: If I admits the strong approximation (9) and if $L_{T} \rightarrow \infty$ as $T \rightarrow \infty$ with $0<L_{T}<$ $T, T / L_{T}$ nondecreasing, $\log \left(T / L_{T}\right) / \log \log T \rightarrow \infty$ and $L_{T} / \log T \rightarrow \infty$, then

$$
\frac{S\left(L_{T}, T, I\right)-\lambda L_{T}}{\beta\left(L_{T}, T\right) \sqrt{\lambda c^{2}}} \rightarrow 1 \text { w.p. } 1 \text { as } T \rightarrow \infty,
$$


where

$$
\beta\left(L_{T}, T\right)=\left(2 L_{T}\left[\log \left(T / L_{T}\right)+\log \log T\right]\right)^{1 / 2}
$$

Proof: Combine Theorem 1.2.1 on p. 30 of [6] with (9), paralleling the proof of Theorem 3.1 .1 on p.115 of [6]. It suffices to work with the Brownian motion because our assumption that $L_{T} / \log T \rightarrow \infty$ as $T \rightarrow \infty$ implies that $(\log T) / \beta\left(L_{T}, T\right) \rightarrow 0$ as $T \rightarrow \infty$.

Note that Theorem 1 remains unchanged if we replace $\beta\left(L_{T}, T\right)$ in (21) by $\left(2 L_{T} \log \left(T / L_{T}\right)\right)^{1 / 2}$, because of our assumption that $\log \left(T / L_{T}\right) / \log \log T \rightarrow \infty$ as $T \rightarrow \infty$. However, $\beta\left(L_{T}, T\right)$ in $(21)$ arises naturally in the proof. Moreover, a partial result holds even when $\log \left(T / L_{T}\right) / \log \log T \rightarrow r, 0<r<\infty$; see Remark 1.2 .2 on page 35 of [6]. Hence, there is a basis for (21) in its current form.

Note that, except for the last term, the approximations for $S$ in (1) comes directly from (20); i.e., we use the approximation $S \approx \lambda L_{T}+\beta\left(L_{T}, T\right) \sqrt{\lambda c^{2}}$. The last term in (1) is added based on our numerical experience with simulations; see Section 6 . It evidently remains to establish a theorem of the form (10) for the sliding window applied to Brownian motion.

To have a more concrete form for approximations, we suppose that $L_{T}=x T^{p}$ for fixed $x$ and $0<p<1$. Note that the conditions on $L_{T}$ in Theorem 1 are satisfied if $L_{T}=x T^{p}$ for any $p$, $0<p<1$, but not for $p=1$, no matter how small $x$ is.

Corollary: If $I$ admits the strong approximation (9) and if $L_{T}=x T^{p}$, for $x>0$ and $0<p<$ 1 , then

$$
\frac{S\left(x T^{p}, T, I\right)-\lambda x T^{p}}{\gamma(x, T) \sqrt{\lambda c^{2}}} \rightarrow 1 \text { w.p. } 1 \text { as } T \rightarrow \infty
$$

where

$$
\gamma(x, T)=\left(2 x T^{p}[(1-p) \log T-\log x+\log \log T]\right)^{1 / 2}
$$

We now obtain a related result for the jumping window $J$ and relate the asymptotics for $J$ and $S$. In particular, we show that they are asymptotically equivalent in this asymptotic regime and provide support for (2).

Theorem 2: Under the assumptions of Theorem 1,

$$
\frac{J\left(L_{T}, T, I\right)-\lambda L_{T}}{b\left(L_{T}, T\right)} \Rightarrow 1 \text { w.p. } 1 \text { as } T \rightarrow \infty
$$

for $b(L, T)$ in (13). If, in addition, $L_{T} /(\log T)^{3} \rightarrow \infty$ as $T \rightarrow \infty$, then

$$
a\left(L_{T}, T\right)\left[J\left(L_{T}, T, I\right)-\lambda L_{T}-b\left(L_{T}, T\right)\right] \Rightarrow Z \text { as } T \rightarrow \infty
$$

for $a(L, T), b(L, T)$ and $Z$ in $(11)-(13)$.

Proof: Combine the strong approximation (9) with the extreme-value result (10). The limit (10) holds as $T \rightarrow \infty$ for fixed $L$, but we need a limit as $T \rightarrow \infty$ and $L \rightarrow \infty$. By the properties of Brownian motion, (10) holds uniformly in $L$ provided that $T / L \rightarrow \infty$; i.e., given that $T$ is an integer multiple of $L,[J(L, T)-\lambda L] / \sqrt{L}$ for Brownian motion with drift $\lambda$ and variance coefficient 1 is distributed the same as $J(1, T / L)$ for Brownian motion with drift 0 and variance coefficient 1. For the case in which $I$ in $J\left(L_{T}, T, I\right)$ is Brownian motion, (22) follows from (10) by dividing by $a\left(L_{T}, T\right) b\left(L_{T}, T\right)$. For the case of a general $I$ in $J\left(L_{T}, T, I\right)$, we apply (9). Under the assumptions of Theorem $1, L_{T} / \log T \rightarrow \infty$, so that $(\log T) / b\left(L_{T}, T\right) \rightarrow 0$ as $T \rightarrow \infty$. For $(23)$, 
we use the additional assumption to obtain $a\left(L_{T}, T\right) \log T \rightarrow 0$ as $T \rightarrow \infty$.

In Theorems 1 and 2 we have provided asymptotics that can serve as the basis for approximations. If better numerical results are desired, especially for smaller values of $T$ and $L$, then the more involved approximation method of Naus [12], [13] is useful.

\section{Discriminating Windows}

The appeal of the asymptotics in $\S 4$ is the simple formulas depending on the process $I$ only via the two parameters $\lambda$ and $c^{2}$. However in some circumstances we may want to learn more about the process $I$ from window processes. For this purpose, it is natural to be guided by $\S 2.4$ of [6], and let

$$
L_{T}=x \log T
$$

For the case of partial sums of i.i.d. random variables, i.e., $I(t)=X_{1}+\cdots+X_{\lfloor t\rfloor}$, where $\lfloor t\rfloor$ is the integer part of $t$,

$$
\frac{S(x \log T, T, I)}{x \log T} \rightarrow \alpha(x) \text { w.p. } 1 \text { as } T \rightarrow \infty,
$$

where

$$
\alpha(x)=\sup \left\{x: \inf _{t} e^{-t x} E e^{t X_{1}} \geq e^{-1 / x}\right\}
$$

see Theorem 2.4 .3 on p. 98 of [6]. Moreover, by Theorem 2.4.5 of [6], the function $\alpha(x)$ in $(26)$ uniquely determines the distribution function of $X_{1}$. More generally, the limit (25) regarded as a function of $x$ seems promising for characterizing the entire process $I$. This seems to be a promising direction for research.

\section{Numerical Comparisons}

In this section we use computer simulation to investigate the approximations in $(1)-(5)$. In all cases we let $\lambda=1$. We start by letting $T=10^{6}$.

We first consider the Poisson process, which has $c^{2}=1$. Tables 2 and 3 display results for $S$ and $J$ based on two experiments, each with 10 independent replications. We let $L$ range from 20 to 5000. Since $T=10^{6}$ and $\log T=13.8$, in this range for $L$ we clearly have $L<<T$, but we only have $\log T<<L$ for larger values of $L$, say $L \geq 100$.

From the first experiment, we saw that the approximation for $S$ provided by Theorem 1 performs reasonably well, but that it can be improved by subtracting the standard deviation in (4). This yields the approximation for $S$ or $E S$ in (1). This refinement was found to help in other experiments as well, so we include it in (1). However, it still remains to develop theoretical justification for this refinement. Note that the standard deviation in (4) is of the same order as $1 / a(L, T)$ in $(10)-(14)$, so that it is natural to anticipate a refinement for $S$ of this form. To confirm this refinement, we conducted the second experiment with different random seeds.

In Table 2 we display the error in the mean of $S$ for each simulation experiment. This is the approximation value (1) minus the simulation estimate. Notice that the approximation for the mean performs well for the entire range of window lengths. Also notice that the estimated errors in the mean are quite a bit smaller than the standard deviation when $L$ is not too small (e.g., for 
$L \geq 100$ ), both predicted by (4) and estimated by simulation. Hence, in the region $\log T<<L$ $<<T$, the approximation is accurate to within the noise, e.g., the maximum of the strong approximation error $O(\log T)=O(13.8)$ and one standard deviation.

Table 3 describes similar results for the jumping window, based on the same simulation experiments. Again, the error in the mean is the approximate value minus the simulation estimate. Here no additional refinement was deemed necessary, so approximation (2) for $J$ follows directly from Theorem 2 and $(10)-(14)$. As with the sliding window, the approximation for the mean performs quite well for a Poisson process over the entire range of window lengths. Again the error in the mean is less than one standard deviation for $L \geq 100$.

From Tables 2 and 3, we see that there is considerable variability in the standard deviation estimates. However, (4) is evidently a reasonable estimate of the standard deviation of both $S$ and $J$.

To test the invariance principle, we also performed the identical simulation experiment for two rate-1 renewal processes with common SCV, $c^{2}=15.4$. One renewal process is an on-off process with a deterministic distance of 0.1 between arrivals during an on period. The on period is geometrically distributed with mean 1 , so that the mean number of arrivals during an on period is 10. As usual, the off period is exponentially distributed. An interarrival time is the mixture of 0.1 and 0.1 plus the exponential off period. The mean of the exponential random variable is chosen so that the mean interarrival time is 1.0. This makes the $\operatorname{SCV} c^{2}=15.4$.

The other renewal process has interarrival times equal to a constant 0.1 plus a hyperexponential $\left(\mathrm{H}_{2}\right)$ random variable, which has a distribution which is a mixture of two exponential distributions; i.e., the $\mathrm{H}_{2}$ distribution has density

$$
f(t)=p \lambda_{1} e^{-\lambda_{1} t}+(1-p) \lambda_{2} e^{-\lambda_{2} t}, t \geq 0 .
$$

The $H_{2}$ distribution is given balanced means, i.e., $p \lambda_{1}^{-1}=(1-p) \lambda_{2}^{-1}$. The remaining two parameters are chosen so that the overall distribution has mean 1 and $\operatorname{SCV} c^{2}=15.4$.

Tables 4 and 5 display the results for these two renewal processes with common SCV $c^{2}=15.4$. Table 4 displays results for the sliding window, while Table 5 displays results for the jumping window. As in Tables 2 and 3, the simulation estimates are based on ten independent replications for each renewal process. A second experiment of ten independent replications yielded similar results.

From Tables 4 and 5, we see that there are significant differences between these two renewal process. However, for larger values of $L$, these differences are relatively small compared to the standard deviation. For $L \leq 500$, though, the differences are dramatic. For larger values of $L$, say $L \geq 500$, the results are roughly consistent with the invariance principle. The approximate mean overestimates the $\left(D+H_{2}\right)$ mean values by about the same amount it underestimates the on-off mean values. The error in the mean relative to the standard deviation decreases significantly as $L$ increases. At about $L \geq 700$, the errors are no more than one standard deviation.

For the smaller values of $L$, we see that the invariance principle is not appropriate. Consistent with $\S 5$, we see that for $L \leq 100$ the two renewal processes behave very differently. Both the means and the standard deviations differ by a factor of two to five in this range. It is interesting that the ratio of the standard deviation estimates of the $\mathrm{D}+\mathrm{H}_{2}$ renewal process to the on-off renewal process increases from 0.22 at $L=20$ to 1.8 for $L=5000$. As with the mean, the approximation falls nicely between the two standard deviation estimates.

We also performed similar experiments with less bursty variants of these same two renewal processes. We found that the approximations performed better when the SCV is decreased from 
15.4 to 4.0. As might be anticipated, this case falls in between the cases $c^{2}=1$ and $c^{2}=15.4$ that we have described in detail.

Next, Table 6 compares the approximation for the ratio $(J-\lambda L) /(S-\lambda L)$ in (3) with simulation estimates for the three renewal processes considered above. Note that the approximation is the same for all three processes. The estimates are the sample means and sample standard deviations of the observed ratio. The average errors over the 14 values with $L \geq 500$ are -0.015 for the Poisson process, -0.001 for the on-off renewal process and -0.027 for the $\left(\mathrm{D}+\mathrm{H}_{2}\right)$-renewal process. Notice that the estimated standard deviations are about 0.09 for the larger $L$ values and about 0.05 for the smaller $L$ values. The estimated errors in the mean are quite small compared to these standard deviation estimates.

Finally, it is interesting to see how the approximations perform for smaller values of $T$ than $10^{6}$. To give an idea, Tables 7 and 8 display results for the sliding and jumping windows, respectively, applied to the Poisson process for $T=10^{3}$ and $T=10^{4}$. Again, the simulation estimates are based on ten independent replications and the error in the mean is the approximation minus the sample mean. Consistent with Tables 2 and 3 , the approximation for the means tend to be slightly low for small values of $L$. Table 7 shows that for $T=10^{4}$ and $L \geq 1000$, where $T / L<10$, the approximation degrades, but it still is within one standard deviation. Table 8 shows that the approximation for the jumping window degrades more for large $L$. The ratio estimates are also highly variable in this region. For example, for $T=10^{4}$ and $L=2.5 \times 10^{3}$, the sample mean and variance of $(J-\lambda L) /(S-\lambda L)$ were 1.19 and 1.83 , respectively. This should not be surprising, since $T / L=4$ in this case. Contrary to the conditions of Theorems 1 and $2, \log (T / L)$ is not large compared to $\log \log T$ in this range, so this degradation in performance can be anticipated.

However, for $L$ neither very small nor very large, Tables 7 and 8 show that the approximations still perform well.

\section{Conclusions}

Our purpose in this paper was to develop simple approximations for the maximum sliding and jumping window content associated with a general input process $I$. Since our intended application is high-speed communication networks, we are interested in relatively long sample paths (very large values of $T$ ) and large window length $L$, with $T$ much larger than $L$. Hence, it is natural to consider applications based on asymptotics.

We proposed approximating the general input process $I$ by a Brownian motion. This approximation depends on the general input process $I$ through only two parameters: its rate $\lambda$ and the limiting value of its index of dispersion, $c^{2}$ in (7). Strong approximations allow us to do this for the entire process yielding an error of $O(\log T)$; see (9).

We then consider extreme-value asymptotics for Brownian motion in order to obtain the basic approximations in (1) and (2). These approximations were supported by Theorems 1 and 2, which established limits as both $T \rightarrow \infty$ and $L \rightarrow \infty$ with $L / \log T \rightarrow \infty$.

We developed the final form of the approximations for $E S$ in (1) and provided additional support for the other approximations by making numerical comparisons with computer simulations. (However, a refinement of Theorem 1 supporting approximation (1) is still needed.) When $T=10^{6}$ and $L=10^{3}$, the basic approximations (1) - (4) perform well. However, when $T=10^{6}$ and $L=50$, and $L$ is no longer much bigger than $\log T$, the simulations confirm that the invariance principle can break down, as predicted by the theory (see §5). This break down was illustrated by the example of the two renewal processes with SCV 15.4. 
Overall, the approximations seem remarkably effective for the parameter range indicated by the theory. Once again, we see that statistical regularity can be captured by appropriate probability limit theorems.

\section{References}

[1] Berger, A.W. and Whitt, W., Comparison of two traffic descriptors for ATM connections in a broadband ISDN, (in preparation).

[2] Bertsekas, D. and Gallager, R., Data Networks, Prentice Hall, Englewood Cliffs, NJ, (1987).

[3] Budka, K.C., Stochastic monotonicity and concavity properties of rate-based flow control mechanisms, IEEE Trans. Aut. Control, 38, to appear (1993).

[4] Cox, D.R. and Lewis P.A.W., The Statistical Analysis of Series of Events, Methuen, London, (1966).

[5] Csörgő, M, Horváth, L., and Steinebach, J., Invariance principles for renewal processes, Ann. Probab, 15, 1441-1460, (1987).

[6] Csörgő, M. and Révész, P., Strong Approximations in Probability and Statistics, Academic Press, New York, (1981).

[7] Erdös, P. and Rényi, A., On a new law of large numbers, J. Analyse Math. 23, 123-131, (1970).

[8] Johnson, N.L. and Kotz, S., Distributions in Statistics: Continuous Univariate Distributions-1, Wiley, New York, (1970).

[9] Komlós, J., Major, P., and Tusnády, G., An approximation of partial sums of independent rv's and the sample D.F-I., Z. Wahrscheinlichkeitsth. Verw, Geb. 32, 111-131, (1975).

[10] Komlós, J., Major, P., and Tusnády, G., An approximation of partial sums of independent random variables and the sample D.F-II., Z. Wahrscheinlichkeitsth. Verw, Geb. 34, 33-58, (1976).

[11] Leadbetter, M.R., Lindgren, G., and Rootzén, H., Extremes and related properties of random sequences and processes, Springer-Verlag, New York (1983).

[12] Naus, J.I., Approximations for distributions of scan statistics, J. Amer. Stat. Assoc. 77, 177-183, (1982).

[13] Naus, J.I., Scan statistics, in Encyclopedia of Statistical Sciences, S. Kotz and N.L. Johnson (eds), 8, 281-284, Wiley, New York, (1988).

[14] Reibman, A.R. and Berger, A.W., Traffic descriptors for VBR video teleconferencing over ATM networks, IEEE/ACM Trans. on Networking, to appear.

[15] Roberts, J., (ed.) Performance Evaluation and Design of Multiservice Networks, COST 224 Final Report, Commission of the European Communities, Luxembourg, (1992). 


\begin{tabular}{|c|c|c|c|c|c|c||}
\hline$L$ & $10^{5}$ & $10^{6}$ & $10^{7}$ & $10^{8}$ & $10^{9}$ & $10^{10}$ \\
\hline 50 & 0.834 & 0.856 & 0.872 & 0.885 & 0.894 & 0.902 \\
\hline 100 & 0.822 & 0.848 & 0.866 & 0.880 & 0.891 & 0.900 \\
\hline 500 & 0.787 & 0.826 & 0.850 & 0.868 & 0.881 & 0.892 \\
\hline 1000 & 0.767 & 0.813 & 0.842 & 0.862 & 0.877 & 0.888 \\
\hline 5000 & 0.700 & 0.777 & 0.819 & 0.846 & 0.865 & 0.879 \\
\hline 10000 & 0.656 & 0.756 & 0.806 & 0.837 & 0.858 & 0.874 \\
\hline
\end{tabular}

Table 1: The estimated ratio $(J-\lambda L) /(S-\lambda L)$ in (3) as a function of $L$ and $T$. 


\begin{tabular}{|c|c|c|c|c|c|c|c|c|}
\hline \multirow{3}{*}{$\begin{array}{c}\text { window } \\
\text { length } \\
L\end{array}$} & \multicolumn{2}{|c|}{$\begin{array}{l}\text { approxi- } \\
\text { mations }\end{array}$} & \multicolumn{6}{|c|}{ simulation estimates } \\
\hline & \multirow{2}{*}{$\begin{array}{c}\text { mean } \\
(1)\end{array}$} & \multirow{2}{*}{$\begin{array}{l}\text { std. } \\
\text { dev. } \\
(4)\end{array}$} & \multicolumn{3}{|c|}{ first experiment } & \multicolumn{3}{|c|}{ second experiment } \\
\hline & & & mean & $\begin{array}{l}\text { std. } \\
\text { dev. }\end{array}$ & $\begin{array}{l}\text { error } \\
\text { in } \\
\text { mean }\end{array}$ & mean & $\begin{array}{l}\text { std. } \\
\text { dev. }\end{array}$ & $\begin{array}{c}\text { error } \\
\text { in } \\
\text { mean }\end{array}$ \\
\hline 20 & 42 & 1.2 & 45 & 1.8 & -3 & 44 & 0.9 & -2 \\
\hline 40 & 70 & 1.8 & 73 & 1.8 & -3 & 72 & 1.6 & -2 \\
\hline 60 & 96 & 2.2 & 99 & 2.4 & -3 & 98 & 2.0 & -2 \\
\hline 80 & 121 & 2.6 & 125 & 2.1 & -4 & 122 & 2.2 & -1 \\
\hline 100 & 146 & 3.0 & 148 & 1.5 & -2 & 147 & 1.8 & -1 \\
\hline 200 & 262 & 4.4 & 262 & 3.2 & 0 & 263 & 4.5 & -1 \\
\hline 300 & 375 & 5.5 & 376 & 3.5 & -1 & 375 & 4.3 & 0 \\
\hline 400 & 485 & 6.5 & 486 & 5.9 & -1 & 484 & 5.0 & 1 \\
\hline 500 & 594 & 7.3 & 594 & 6.3 & 0 & 591 & 5.6 & 3 \\
\hline 600 & 702 & 8.1 & 703 & 8.1 & -1 & 697 & 7.2 & 5 \\
\hline 700 & 809 & 8.9 & 809 & 8.2 & 0 & 802 & 7.3 & 7 \\
\hline 800 & 915 & 9.6 & 918 & 11.6 & -3 & 910 & 10.2 & 5 \\
\hline 900 & 1021 & 10.3 & 1024 & 8.8 & -3 & 1016 & 9.6 & 5 \\
\hline 1000 & 1127 & 10.9 & 1128 & 8.6 & -1 & 1118 & 6.9 & 9 \\
\hline 1500 & 1652 & 13.7 & 1654 & 22.3 & -2 & 1645 & 10.5 & 7 \\
\hline 2000 & 2172 & 16.2 & 2173 & 23.6 & -1 & 2164 & 12.7 & 8 \\
\hline 2500 & 2689 & 18.5 & 2692 & 29.6 & -3 & 2685 & 21.1 & 4 \\
\hline 3000 & 3204 & 20.6 & 3206 & 22.5 & -2 & 3199 & 24.9 & 5 \\
\hline 3500 & 3718 & 22.5 & 3718 & 22.2 & 0 & 3721 & 26.6 & -3 \\
\hline 4000 & 4231 & 24.4 & 4223 & 19.0 & 8 & 4228 & 29.1 & 3 \\
\hline 4500 & 4743 & 26.1 & 4732 & 15.4 & 11 & 4734 & 38.2 & 9 \\
\hline 5000 & 5254 & 27.8 & 5247 & 19.7 & 7 & 5237 & 35.9 & 17 \\
\hline
\end{tabular}

Table 2. A comparison of approximations with simulation estimates for the mean and standard deviation of the maximum sliding window content of a rate-1 Poisson process for a total time period $T=10^{6}$ as a function of the window length $L$. The simulation estimates are based on two experiments, each of ten independent replications. 


\begin{tabular}{|c|c|c|c|c|c|c|c|c|}
\hline \multirow{3}{*}{$\begin{array}{c}\text { window } \\
\text { length } \\
L\end{array}$} & \multicolumn{2}{|c|}{$\begin{array}{l}\text { approxi- } \\
\text { mations }\end{array}$} & \multicolumn{6}{|c|}{ simulation estimates } \\
\hline & \multirow{2}{*}{$\begin{array}{c}\text { mean } \\
(2)\end{array}$} & \multirow{2}{*}{$\begin{array}{l}\text { std. } \\
\text { dev. } \\
(4)\end{array}$} & \multicolumn{3}{|c|}{ first experiment } & \multicolumn{3}{|c|}{ second experiment } \\
\hline & & & mean & $\begin{array}{l}\text { std. } \\
\text { dev. }\end{array}$ & $\begin{array}{c}\text { error in } \\
\text { mean }\end{array}$ & mean & std. dev. & $\begin{array}{c}\text { error in } \\
\text { mean }\end{array}$ \\
\hline 20 & 39 & 1.2 & 42 & 1.9 & -3 & 41 & 0.9 & -2 \\
\hline 40 & 66 & 1.8 & 69 & 2.9 & -3 & 68 & 1.6 & -2 \\
\hline 60 & 91 & 2.2 & 93 & 2.9 & -2 & 93 & 2.2 & -2 \\
\hline 80 & 115 & 2.6 & 117 & 2.6 & -2 & 117 & 2.3 & -2 \\
\hline 100 & 139 & 3.0 & 141 & 3.5 & -2 & 141 & 4.3 & -2 \\
\hline 200 & 252 & 4.4 & 253 & 4.4 & -1 & 251 & 4.3 & 1 \\
\hline 300 & 362 & 5.5 & 364 & 4.6 & -2 & 365 & 5.0 & -3 \\
\hline 400 & 470 & 6.5 & 474 & 9.4 & -4 & 471 & 4.2 & -1 \\
\hline 500 & 577 & 7.3 & 579 & 6.6 & -2 & 575 & 6.1 & 2 \\
\hline 600 & 684 & 8.1 & 688 & 7.7 & -4 & 684 & 6.1 & 0 \\
\hline 700 & 789 & 8.9 & 793 & 8.9 & -4 & 785 & 6.9 & 4 \\
\hline 800 & 894 & 9.6 & 896 & 8.9 & -2 & 887 & 6.8 & 7 \\
\hline 900 & 999 & 10.3 & 1001 & 10.4 & -2 & 999 & 8.4 & 0 \\
\hline 1000 & 1103 & 10.9 & 1100 & 12.0 & 3 & 1097 & 9.0 & 6 \\
\hline 1500 & 1622 & 13.7 & 1624 & 15.7 & -2 & 1627 & 14.2 & -5 \\
\hline 2000 & 2137 & 16.2 & 2134 & 19.4 & 3 & 2133 & 17.2 & 4 \\
\hline 2500 & 2650 & 18.5 & 2661 & 31.3 & -11 & 2654 & 12.7 & -4 \\
\hline 3000 & 3161 & 20.6 & 3164 & 25.8 & -3 & 3167 & 27.3 & -6 \\
\hline 3500 & 3672 & 22.5 & 3669 & 23.9 & 3 & 3663 & 21.2 & 9 \\
\hline 4000 & 4181 & 24.4 & 4168 & 19.0 & 13 & 4181 & 17.0 & 0 \\
\hline 4500 & 4689 & 26.1 & 4696 & 24.7 & -7 & 4684 & 31.7 & 5 \\
\hline 5000 & 5197 & 27.8 & 5181 & 26.1 & 16 & 5178 & 18.1 & 19 \\
\hline
\end{tabular}

Table 3. A comparison of approximations with simulation estimate for the mean and standard deviation of the maximum jumping window content of a rate-1 Poisson process for a total time period $T=10^{6}$ as a function of the window length $L$. The simulation estimates are based on two experiments, each of ten independent replications. 


\begin{tabular}{|c|c|c|c|c|c|c|c|c|}
\hline \multirow{3}{*}{$\begin{array}{c}\text { window } \\
\text { length } \\
L\end{array}$} & \multirow{2}{*}{\multicolumn{2}{|c|}{ approximations }} & \multicolumn{6}{|c|}{ simulation estimates } \\
\hline & & & \multicolumn{2}{|c|}{ mean } & \multicolumn{2}{|c|}{ error in mean } & \multicolumn{2}{|c|}{ std. dev. } \\
\hline & $\begin{array}{c}\text { mean } \\
(1)\end{array}$ & $\begin{array}{l}\text { std. dev. } \\
\text { (4) }\end{array}$ & on-off & $\mathrm{D}+\mathrm{H}_{2}$ & on-off & $\mathrm{D}+\mathrm{H}_{2}$ & on-off & $D+H_{2}$ \\
\hline 20 & 106 & 4.8 & 152 & 58 & -46 & 48 & 7.3 & 1.6 \\
\hline 40 & 158 & 7.1 & 202 & 101 & -44 & 57 & 7.7 & 3.0 \\
\hline 60 & 202 & 8.8 & 251 & 140 & -49 & 62 & 9.6 & 2.9 \\
\hline 80 & 242 & 10.3 & 287 & 179 & -45 & 63 & 21.3 & 2.9 \\
\hline 100 & 279 & 11.7 & 330 & 214 & -51 & 65 & 19.0 & 3.7 \\
\hline 200 & 445 & 17.2 & 483 & 380 & -38 & 65 & 10.5 & 7.0 \\
\hline 300 & 593 & 21.6 & 645 & 531 & -52 & 62 & 36.3 & 19.2 \\
\hline 400 & 733 & 25.4 & 774 & 674 & -41 & 59 & 31.4 & 20.1 \\
\hline 500 & 868 & 28.8 & 919 & 816 & -51 & 52 & 38.7 & 16.4 \\
\hline 600 & 999 & 31.9 & 1035 & 951 & -36 & 48 & 40.6 & 14.5 \\
\hline 700 & 1127 & 34.9 & 1157 & 1078 & -30 & 49 & 42.2 & 16.5 \\
\hline 800 & 1252 & 37.6 & 1287 & 1197 & -35 & 55 & 35.4 & 19.0 \\
\hline 900 & 1376 & 40.2 & 1418 & 1327 & -42 & 49 & 43.0 & 26.9 \\
\hline 1000 & 1499 & 42.7 & 1542 & 1454 & -43 & 45 & 51.6 & 27.4 \\
\hline 1500 & 2095 & 53.9 & 2134 & 2037 & -39 & 58 & 53.9 & 29.6 \\
\hline 2000 & 2674 & 63.7 & 2691 & 2650 & -17 & 24 & 55.7 & 34.7 \\
\hline 2500 & 3242 & 72.5 & 3275 & 3209 & -33 & 33 & 57.9 & 75.3 \\
\hline 3000 & 3802 & 80.7 & 3829 & 3799 & -27 & 3 & 67.1 & 92.9 \\
\hline 3500 & 4356 & 88.3 & 4398 & 4332 & -42 & 24 & 48.4 & 110.0 \\
\hline 4000 & 4906 & 95.6 & 4943 & 4851 & -37 & 55 & 88.4 & 135.3 \\
\hline 4500 & 5452 & 102.5 & 5471 & 5410 & -19 & 42 & 92.5 & 144.2 \\
\hline 5000 & 5995 & 109.1 & 6008 & 5966 & -13 & 29 & 80.9 & 145.4 \\
\hline
\end{tabular}

Table 4. A comparison of approximations with simulation estimates for the mean and standard deviation of the maximum sliding window content of two rate- 1 renewal processes with SCV $\mathrm{c}^{2}=15.4$ for a total time period of length $T=10^{6}$ as a function of the window length $L$. The simulation estimates are based on ten independent replications. 


\begin{tabular}{|c|c|c|c|c|c|c|c|c|}
\hline \multirow{3}{*}{$\begin{array}{c}\text { window } \\
\text { length } \\
L\end{array}$} & \multirow{2}{*}{\multicolumn{2}{|c|}{ approximations }} & \multicolumn{6}{|c|}{ simulation estimates } \\
\hline & & & \multicolumn{2}{|c|}{ mean } & \multicolumn{2}{|c|}{ error in mean } & \multicolumn{2}{|c|}{ std. dev. } \\
\hline & $\begin{array}{c}\text { mean } \\
(2)\end{array}$ & $\begin{array}{l}\text { std. dev. } \\
\text { (4) }\end{array}$ & on-off & $\mathrm{D}+\mathrm{H}_{2}$ & on-off & $\mathrm{D}+\mathrm{H}_{2}$ & on-off & $\mathrm{D}+\mathrm{H}_{2}$ \\
\hline 20 & 95 & 4.8 & 135 & 55 & -40 & 40 & 8.2 & 2.2 \\
\hline 40 & 141 & 7.1 & 186 & 96 & -45 & 45 & 8.8 & 2.8 \\
\hline 60 & 181 & 8.8 & 227 & 135 & -46 & 46 & 11.4 & 3.0 \\
\hline 80 & 218 & 10.3 & 257 & 170 & -39 & 48 & 13.0 & 2.9 \\
\hline 100 & 252 & 11.7 & 306 & 203 & -54 & 49 & 21.9 & 4.3 \\
\hline 200 & 405 & 17.2 & 448 & 362 & -43 & 43 & 22.8 & 10.1 \\
\hline 300 & 544 & 21.6 & 591 & 507 & -47 & 37 & 24.4 & 15.2 \\
\hline 400 & 676 & 25.4 & 728 & 647 & -52 & 29 & 33.5 & 19.2 \\
\hline 500 & 804 & 28.8 & 847 & 771 & -43 & 33 & 22.9 & 15.6 \\
\hline 600 & 928 & 31.9 & 958 & 897 & -30 & 31 & 36.3 & 27.9 \\
\hline 700 & 1050 & 34.9 & 1077 & 1022 & -27 & 28 & 29.7 & 25.0 \\
\hline 800 & 1170 & 37.6 & 1206 & 1151 & -36 & 19 & 46.1 & 29.0 \\
\hline 900 & 1289 & 40.2 & 1318 & 1263 & -29 & 26 & 39.1 & 24.2 \\
\hline 1000 & 1406 & 42.7 & 1434 & 1388 & -28 & 18 & 34.4 & 32.5 \\
\hline 1500 & 1980 & 53.9 & 1991 & 1954 & -11 & 26 & 59.2 & 20.6 \\
\hline 2000 & 2539 & 63.7 & 2566 & 2518 & -27 & 21 & 65.7 & 82.1 \\
\hline 2500 & 3089 & 72.5 & 3091 & 3070 & -2 & 19 & 49.0 & 76.0 \\
\hline 3000 & 3634 & 80.7 & 3644 & 3665 & -10 & -31 & 58.2 & 116.5 \\
\hline 3500 & 4173 & 88.3 & 4170 & 4138 & 3 & 35 & 60.6 & 80.6 \\
\hline 4000 & 4709 & 95.6 & 4761 & 4673 & -52 & 36 & 61.2 & 103.0 \\
\hline 4500 & 5243 & 102.5 & 5283 & 5228 & -40 & 15 & 59.6 & 142.0 \\
\hline 5000 & 5773 & 109.1 & 5801 & 5774 & -28 & -1 & 117.7 & 109.4 \\
\hline
\end{tabular}

Table 5. A comparison of approximations with simulation estimates for the mean and standard deviation of the maximum jumping window content of two rate- 1 renewal processes with SCV c ${ }^{2}=15.4$ for a total time period of length $T=10^{6}$ as a function of the window length $L$. The simulation estimates are based on ten independent replications. 


\begin{tabular}{|c|c|c|c|c|c|c|c|c|c|c|}
\hline \multirow{3}{*}{$\begin{array}{c}\text { window } \\
\text { length } \\
L\end{array}$} & \multirow{3}{*}{$\begin{array}{l}\text { app- } \\
\text { rox. } \\
\text { ratio } \\
(3)\end{array}$} & \multicolumn{9}{|c|}{ simulation estimates } \\
\hline & & \multicolumn{3}{|c|}{ Poisson } & \multicolumn{3}{|c|}{ on-off, $c^{2}=15.4$} & \multicolumn{3}{|c|}{$D+H_{2}, \mathrm{c}^{2}=15.4$} \\
\hline & & $\begin{array}{l}\mathrm{m} \\
\mathrm{e} \\
\mathrm{a} \\
\mathrm{n}\end{array}$ & $\begin{array}{l}\text { std. } \\
\text { dev. }\end{array}$ & $\begin{array}{l}\mathrm{e} \\
\mathrm{r} \\
\mathrm{r} \\
\mathrm{o} \\
\mathrm{r}\end{array}$ & $\begin{array}{l}\mathrm{m} \\
\mathrm{e} \\
\mathrm{a} \\
\mathrm{n}\end{array}$ & $\begin{array}{l}\text { std } \\
\text { dev }\end{array}$ & $\begin{array}{l}\mathrm{e} \\
\mathrm{r} \\
\mathrm{r} \\
\mathrm{o} \\
\mathrm{r}\end{array}$ & $\begin{array}{c}\mathrm{m} \\
\mathrm{e} \\
\mathrm{a} \\
\mathrm{n}\end{array}$ & $\begin{array}{l}\text { std. } \\
\text { dev. }\end{array}$ & $\begin{array}{l}\mathrm{e} \\
\mathrm{r} \\
\mathrm{r} \\
\mathrm{o} \\
\mathrm{r}\end{array}$ \\
\hline 20 & .865 & .856 & .050 & .009 & .878 & .073 & -.013 & .922 & .050 & -.057 \\
\hline 40 & .858 & 871 & .045 & -.013 & .904 & .076 & -.046 & .928 & .042 & -.070 \\
\hline 60 & .854 & .871 & .058 & -.017 & .878 & .067 & -.024 & .936 & .046 & -.082 \\
\hline 80 & .851 & .883 & .050 & -.032 & .862 & .096 & -.011 & .912 & .033 & -.061 \\
\hline 100 & .848 & .885 & .075 & -.037 & .900 & .071 & -.052 & .897 & .030 & -.049 \\
\hline 200 & .839 & .816 & .086 & .023 & .876 & .075 & -.037 & .903 & .028 & -.064 \\
\hline 300 & .833 & .870 & .055 & -.037 & .848 & .084 & -.015 & .899 & .040 & -.066 \\
\hline 400 & .829 & .845 & .060 & -.016 & .878 & .062 & -.049 & .902 & .054 & -.073 \\
\hline 500 & .826 & .826 & .080 & .000 & .831 & .056 & -.005 & .859 & .041 & -.033 \\
\hline 600 & .823 & .871 & .089 & -.048 & .827 & .093 & -.004 & .844 & .070 & -.021 \\
\hline 700 & .820 & .832 & .093 & -.012 & .828 & .049 & -.008 & .851 & .069 & -.031 \\
\hline 800 & .818 & .798 & .079 & .020 & .833 & .067 & -.015 & .884 & .068 & -.066 \\
\hline 900 & .815 & .862 & .084 & -.047 & .812 & .094 & .003 & .854 & .088 & -.039 \\
\hline 1000 & .813 & .827 & .088 & -.014 & .804 & .074 & .009 & .856 & .085 & -.043 \\
\hline 1500 & .806 & .881 & .085 & -.075 & .775 & 080 & .031 & .848 & .066 & -.042 \\
\hline 2000 & .799 & .814 & .074 & -.015 & .822 & .095 & -.023 & .795 & .103 & .004 \\
\hline 2500 & .794 & .843 & .108 & -.049 & .765 & .079 & .029 & .802 & .040 & -.008 \\
\hline 3000 & .790 & .841 & .090 & -.051 & .781 & .100 & .009 & .831 & .090 & -.041 \\
\hline 3500 & .786 & .742 & .070 & .044 & .748 & 079 & .038 & .772 & .094 & .014 \\
\hline 4000 & .783 & .803 & .082 & -.020 & .811 & .085 & -.028 & .794 & .072 & -.011 \\
\hline 4500 & .780 & .791 & .102 & -.011 & .813 & .094 & -.033 & .801 & .091 & -.021 \\
\hline 5000 & .777 & .760 & .104 & .017 & .794 & .090 & -.017 & .811 & .122 & -.034 \\
\hline \multicolumn{4}{|c|}{$\begin{array}{l}\text { average errors for } \\
\qquad L \geq 500\end{array}$} & -.015 & & & -.001 & & & -.027 \\
\hline
\end{tabular}

Table 6. A comparison of the approximation for the normalized ratio $(J-\lambda L) /(S-\lambda L)$ with simulation estimates for the Poisson process, the on-off renewal process, and the $D+H_{2}$ renewal process for a time period $T=10^{6}$ as a function of the window length $L$. The simulation estimates are based on ten independent replications. The error is the approximation minus the estimated mean. 


\begin{tabular}{|c|c|c|c|c|c|c|c|c|}
\hline \multirow{3}{*}{$\begin{array}{c}\text { window } \\
\text { length } \\
L\end{array}$} & \multicolumn{4}{|c|}{$\mathrm{T}=10^{3}$} & \multicolumn{4}{|c|}{$\mathrm{T}=10^{4}$} \\
\hline & \multirow{2}{*}{$\begin{array}{c}\text { approx. } \\
\text { mean (1) }\end{array}$} & \multicolumn{3}{|c|}{ simulation estimates } & \multirow{2}{*}{$\begin{array}{c}\text { approx. } \\
\text { mean } \\
(1)\end{array}$} & \multicolumn{3}{|c|}{ simulation estimates } \\
\hline & & mean & $\begin{array}{l}\text { error } \\
\text { in } \\
\text { mean }\end{array}$ & $\begin{array}{l}\text { std. } \\
\text { dev. }\end{array}$ & & mean & $\begin{array}{l}\text { error } \\
\text { in } \\
\text { mean }\end{array}$ & $\begin{array}{l}\text { std. } \\
\text { dev. }\end{array}$ \\
\hline 10 & 20 & 20.4 & -0.4 & 1.5 & 22 & 24.9 & -2.9 & 1.6 \\
\hline 20 & 33 & 34.3 & -1.3 & 2.1 & 37 & 38.3 & -1.3 & 2.3 \\
\hline 40 & 57 & 57.5 & -0.5 & 2.5 & 62 & 62.8 & -0.8 & 2.3 \\
\hline 60 & 80 & 78.6 & 1.4 & 2.6 & 87 & 86.6 & 0.4 & 5.1 \\
\hline 80 & 102 & 101.7 & 0.3 & 3.3 & 110 & 110.6 & -0.6 & 6.9 \\
\hline 100 & 123 & 122.4 & 0.6 & 4.5 & 133 & 132.8 & 0.2 & 6.9 \\
\hline 200 & 228 & 228.1 & -0.1 & 9.2 & 243 & 241.3 & 1.7 & 7.8 \\
\hline 300 & 329 & 328.1 & 0.9 & 11.2 & 350 & 347.5 & 2.5 & 12.5 \\
\hline 400 & 429 & 427.1 & 1.9 & 13.9 & 456 & 452.2 & 3.8 & 13.9 \\
\hline 500 & 527 & 526.4 & 0.6 & 17.9 & 561 & 558.5 & 2.5 & 16.1 \\
\hline 1000 & & & & & 1076 & 1062.2 & 13.8 & 22.5 \\
\hline 2000 & & & & & 2092 & 2065.2 & 26.8 & 34.9 \\
\hline 4000 & & & & & 4099 & 4054.7 & 44.3 & 56.7 \\
\hline 5000 & & & & & 5094 & 5048.2 & 45.8 & 64.5 \\
\hline
\end{tabular}

Table 7. A comparison of approximations with simulation estimate for the mean of the maximum sliding window content of a rate- 1 Poisson process for total time periods $T=10^{3}$ and $T=10^{4}$ as a function of the window length $L$. The simulation estimates are based on ten independent replications. 


\begin{tabular}{|c|c|c|c|c|c|c|c|c|}
\hline \multirow{3}{*}{$\begin{array}{c}\text { window } \\
\text { length } \\
L\end{array}$} & \multicolumn{4}{|c|}{$\mathrm{T}=10^{3}$} & \multicolumn{4}{|c|}{$\mathrm{T}=10^{4}$} \\
\hline & \multirow{2}{*}{$\begin{array}{c}\text { app- } \\
\text { rox. } \\
\begin{array}{c}\text { mean } \\
(2)\end{array}\end{array}$} & \multicolumn{3}{|c|}{ simulation estimates } & \multirow{2}{*}{$\begin{array}{c}\begin{array}{c}\text { app- } \\
\text { rox. }\end{array} \\
\begin{array}{c}\text { mean } \\
(2)\end{array}\end{array}$} & \multicolumn{3}{|c|}{ simulation estimates } \\
\hline & & mean & $\begin{array}{l}\text { error } \\
\text { in } \\
\text { mean }\end{array}$ & $\begin{array}{l}\text { std. } \\
\text { dev. }\end{array}$ & & mean & $\begin{array}{l}\text { error } \\
\text { in } \\
\text { mean }\end{array}$ & $\begin{array}{l}\text { std. } \\
\text { dev. }\end{array}$ \\
\hline 10 & 18 & 18.8 & -0.8 & 1.2 & 20 & 21.4 & -1.4 & 1.6 \\
\hline 20 & 30 & 30.5 & -0.5 & 2.2 & 34 & 34.5 & -0.5 & 1.3 \\
\hline 40 & 53 & 52.3 & 0.7 & 3.3 & 58 & 58.1 & -0.1 & 1.9 \\
\hline 60 & 74 & 73.3 & 0.7 & 3.8 & 81 & 79.9 & 1.1 & 3.6 \\
\hline 80 & 96 & 94.8 & 1.2 & 4.5 & 104 & 102.8 & 1.2 & 5.1 \\
\hline 100 & 116 & 113.6 & 2.4 & 5.2 & 126 & 124.8 & 1.2 & 3.8 \\
\hline 200 & 218 & 216.1 & 1.9 & 9.0 & 233 & 230.2 & 2.8 & 7.8 \\
\hline 300 & 318 & 314.4 & 3.6 & 12.7 & 337 & 335.4 & 1.6 & 13.4 \\
\hline 400 & 418 & 411.7 & 6.3 & 14.0 & 441 & 437.3 & 3.7 & 18.2 \\
\hline 500 & 517 & 504.4 & 12.6 & 14.1 & 543 & 542.9 & 0.1 & 17.3 \\
\hline 1000 & & & & & 1052 & 1042.3 & 9.7 & 24.8 \\
\hline 2000 & & & & & 2057 & 2047.6 & $9: 4$ & 33.1 \\
\hline 4000 & & & & & 4056 & 4018.4 & 37.6 & 39.2 \\
\hline 5000 & & & & & 5053 & 5013.9 & 39.1 & 59.0 \\
\hline
\end{tabular}

Table 8. A comparison of approximations with simulation estimates for the mean of the maximum jumping window content of a rate-1 Poisson process for total time periods $T=10^{3}$ and $T=10^{4}$ as a function of the window length $L$. The simulation estimates are based on ten independent replications. 


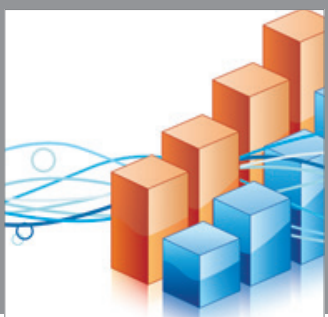

Advances in

Operations Research

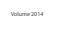

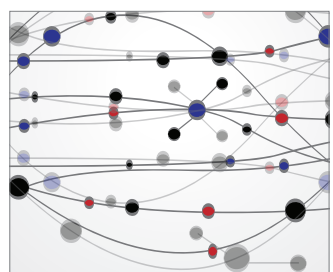

\section{The Scientific} World Journal
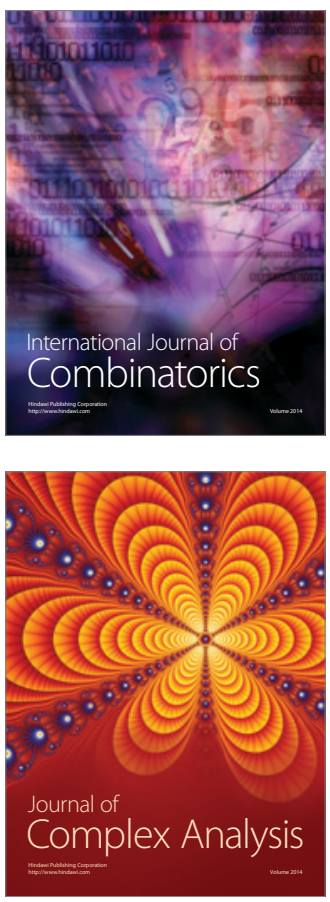

International Journal of

Mathematics and

Mathematical

Sciences
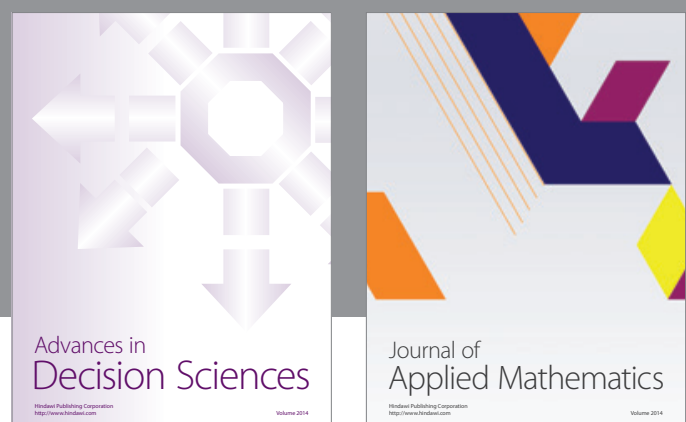

Journal of

Applied Mathematics
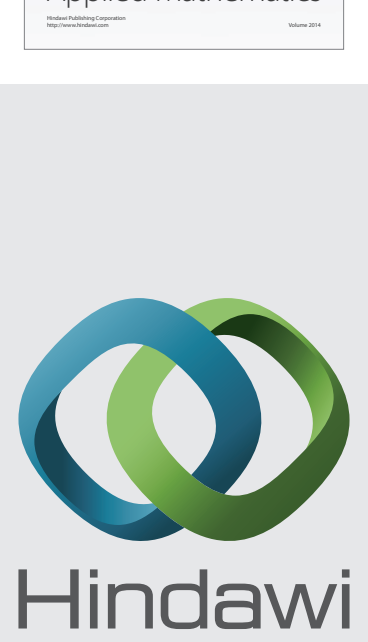

Submit your manuscripts at http://www.hindawi.com
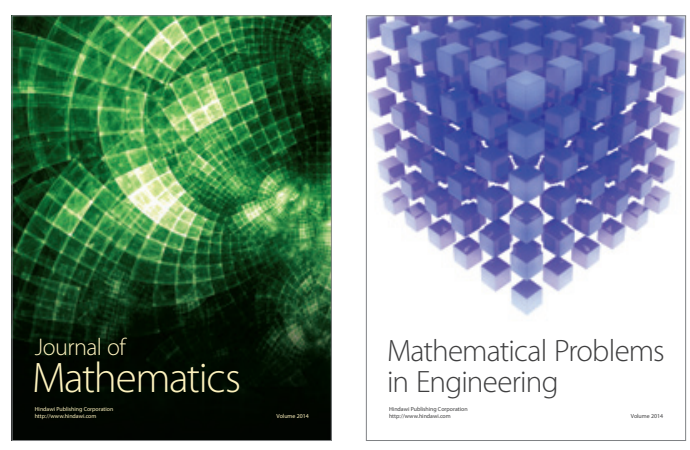

Mathematical Problems in Engineering
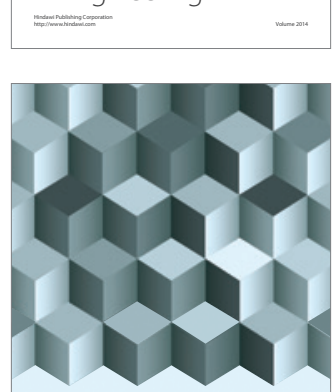

Journal of

Function Spaces
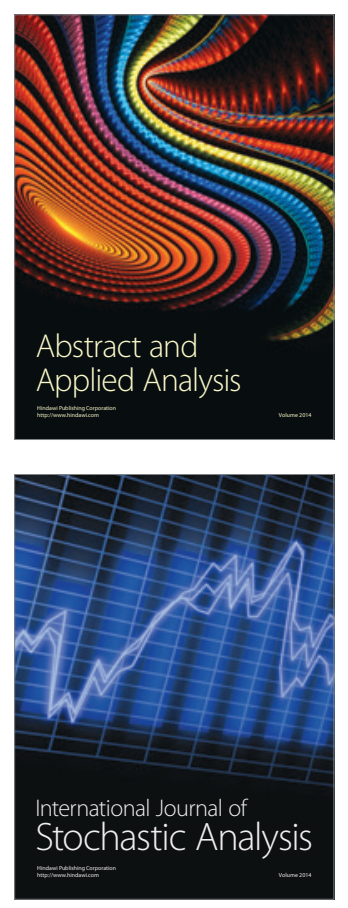

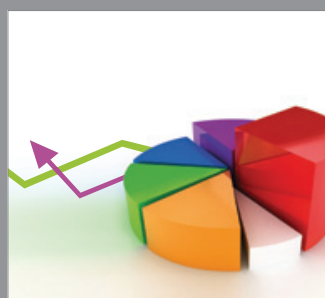

ournal of

Probability and Statistics

Promensencen
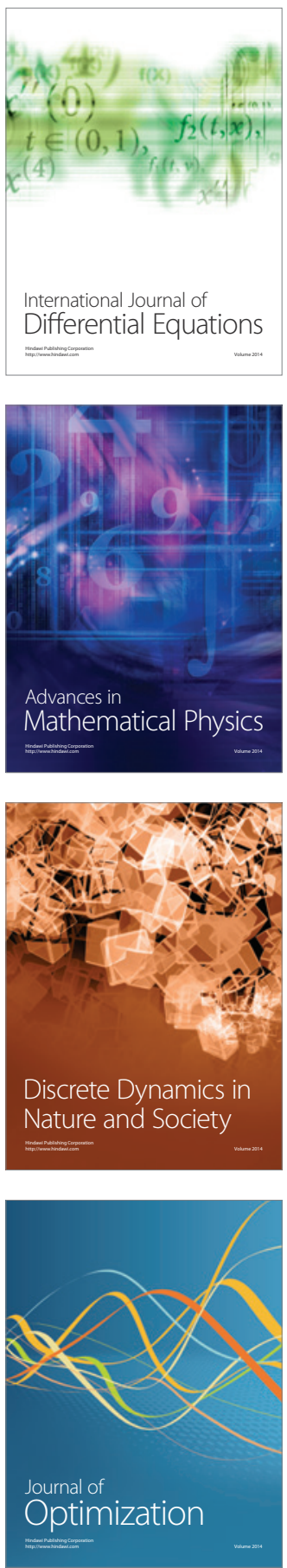